\title{
Ética de las organizaciones de Servicios Sociales (parte I)
}

\author{
Ethics of Social Service organizations (part I) \\ Xavier Pelegri ViañA \\ Universitat de Lleida \\ xpelegri@geosoc.udl.cat
}

Recibido: $05 / 11 / 2012$

Revisado: $16 / 11 / 2012$

Aceptado: 28/02/2013

Disponible on line: 16/04/2013

Una sociedad decente es aquella en que las instituciones no humillan a las personas.

Avishai Margalit (1997)

\begin{abstract}
Resumen
Este artículo, por su extensión, se compone de dos partes y en su conjunto trata de establecer la conveniencia de introducir o profundizar la reflexión ética en las organizaciones de Servicios Sociales. Esta primera parte se dedica a descubrir las características de las organizaciones del ámbito del bienestar y cómo incide en ellas la dimensión colectiva de la ética. Así avanzamos, a partir de preguntas esenciales, hacia las razones que exigen un mayor rigor analítico y una praxis más consecuente de las entidades en las que trabajamos o con las que trabajamos los profesionales de intervención social. Aunque se trata de la parte más general, se abordan aquellos elementos necesarios para enmarcar la segunda parte en la que se propondrán conclusiones concretas para la inclusión de la dimensión ética en los Servicios Sociales.

Palabras clave: ética, organizaciones, Servicios Sociales, profesionales, Trabajo Social.
\end{abstract}

\begin{abstract}
Due to its length, this article is composed of two parts and as a whole it seeks to establish whether to introduce or deepen ethical reflection in social service organizations. This first part is devoted to discovering the characteristics of the organizations in the area of social welfare and how the collective dimension of ethics impacts on them. Then, starting with a set of essential questions, we move to the reasons that require greater analytical rigour and a more consistent practice of the entities for which (or with which) the social intervention professionals work. Although this is the most general part, those elements necessary to frame the second part are addressed. Specific conclusions for the inclusion of the ethical dimension in social services will be proposed in the second part.
\end{abstract}

Keywords: ethics, organizations, social services, professionals, social work.

Referencia normalizada: Pelegri Viaña, X. (2013): «Ética de las organizaciones de Servicios Sociales (parte I)». Cuadernos de Trabajo Social, 26(1): 139-148.

Sumario: (Parte I): Introducción. 1. Encuadre del tema. 2. Las organizaciones de Servicios Sociales. 3. Particularidades de la ética organizacional. (Parte II): 4. Práctica ética de las organizaciones de Servicios Sociales. 5. Compromiso del profesional. 6. Referencias bibliográficas.

\section{Introducción}

En los últimos años hemos asistido a un cierto auge de los temas relacionados con la ética en general y, más concretamente, de la ética de las profesiones. Dentro de esta última, no han quedado atrás los aportes realizados desde la profesión y para la profesión de Trabajo Social, así como las cuestiones, problemas y dilemas que se le plantean en los variados contextos de intervención social. De ello dan testimonio los libros y artículos que se han publicado, las conferencias y seminarios impartidos y, como summum, la existencia de estructuras relativamente estables de reflexión y formación como son el Comité d'Ética de serveis socials de Catalunya, el Observatori d'Ética aplicada a la 
Intervenció Social y el Máster en Ètica Aplicada a la Intervenció Social, Psicoeducativa i Sociosanitària que imparte el mismo Observatorio.

En el marco de dicha proliferación, no obstante, no hemos encontrado muchas referencias teóricas que incidan en las entidades donde los profesionales de Servicios Sociales desarrollan su actividad. Incluso, cuando éstas aparecen, lo hacen como telón de fondo del verdadero leitmotiv que casi siempre es la ética de sus empleados o profesionales. Si queremos encontrar un cierto discurso sobre la ética organizacional lo habremos de buscar en otros campos de actividad: bien en sectores del Estado de bienestar (la sanidad, por ejemplo, que tiene muchos años de experiencia) o, de forma más genérica, en el ámbito de la Administración Pública o en el de la empresa, con el filón de la llamada «responsabilidad social empresarial» o corporativa.

Por consiguiente, después de haber revisado los materiales disponibles, me propongo dar un pequeño paso y empezar a cubrir esta laguna. Me interesa básicamente centrar la reflexión en el comportamiento de las organizaciones sociales $\mathrm{y}$, en concreto, de las organizaciones que prestan servicios sociales, puesto que son las que concentran la mayoría de profesionales de la intervención social. Aunque son complementarias, trataré más de la ética de las organizaciones que de la ética en las organizaciones. Por lo tanto, obviaré, siempre que no sea imprescindible, las disertaciones sobre lo que concierne a la ética profesional del trabajador social o de otras profesiones que se ejercen en los servicios sociales, puesto que ya han sido convenientemente tratadas por muchos autores.

Considero que la ética de las organizaciones no solo debe interesar a los directivos o máximos responsables de las diferentes entidades, sino también a los profesionales y a los empleados de base que trabajan en ellas. Aplicar la ética es imprimir una especie de pátina a todos los actos que generan los miembros en nombre de la organización; por lo tanto, es una dimensión más de la gestión de lo social, entendiendo la gestión - como Veronica Coulshed (1998) - que «todos los trabajadores so- ciales [tómese aquí en un sentido amplio] realizan tareas de gestión: nuestros círculos de actividad se extienden desde la gestión de uno mismo a la gestión de los demás y después a la gestión de los sistemas» (Coulshed, 1998, p. 20).

Utilizaré el concepto organización diferenciándolo del de institución aunque no haya un total consenso en sus significados y, a menudo, en el lenguaje cotidiano tiendan a confundirse. Una institución, en su concepción más simple y genérica, es una convención (Douglas, 1996); pero aquí, las instituciones sociales nos interesan en la medida que «son núcleos básicos de la organización social comunes a todas las sociedades y encargadas de solventar algunos de sus problemas fundamentales») (Valero, 2008, p. 21). En cambio, entiendo que una organización es una asociación de personas que tienen unos objetivos determinados y que funciona según un esquema convenido y una estructura de relación entre roles. Por lo tanto, mientras que «las instituciones no nacen de la acción deliberada, las organizaciones son deliberadas y creadas a través de instrumentos formales»» (p. 25) ${ }^{1}$.

\section{Encuadre del tema}

Una primera pregunta que nos planteamos para enfocar estas disertaciones es si la ética - es decir, la reflexión sobre lo que es moral- puede ser atribuida a una organización. ¿Tiene esta construcción social los atributos necesarios para hacer una evaluación moral? ¿No son los valores, los principios o los dilemas éticos, atributos exclusivos de las personas físicas que son las que tienen per se capacidad de discernir? En consecuencia, ¿no estaremos atribuyendo a las organizaciones la responsabilidad de unos comportamientos que sólo corresponden a sus miembros, sean estos dirigentes o empleados?

Es cierto que la mayoría de las veces nos encontramos que la reflexión ética se ha centrado casi exclusivamente en los profesionales. Incluso cuando se trae a colación la organización, en muchos casos, se habla más del comportamiento moral de los empleados en su organización que de cómo ésta en su conjunto

\footnotetext{
1 Aunque aquí adoptamos este enfoque, hay que advertir que en el ámbito de los servicios sociales en muchas ocasiones a las organizaciones — sobre todo si son administraciones públicas — se las denomina «instituciones», y así aparece en algunas citas.
} 
debería comportarse moralmente. Por mi parte suscribo lo que dice Lozano: «no basta que haya una actuación ética por parte del profesional y de cada persona que trabaja en la organización; es necesario que exista una ética de la organización» (citado en Hortal, 2002, p. 73). Estos autores afirman que la ética en las organizaciones y la ética de las organizaciones no son incompatibles, sino que tienen lógicas complementarias. La ética tiene, pues, que considerar tanto lo que cada persona ha de hacer en su trabajo como aquello que debe hacer la organización para que el trabajo colectivo responda a los valores que propugna. Refiriéndose a la empresa, pero siendo perfectamente extrapolable a todas las organizaciones, Adela Cortina (1997) dice «no [es] que la responsabilidad de los individuos se diluya en la del conjunto de la empresa, sino que la ética no es solo individual, sino también corporativa y comunitaria» (p. 270).

Esta reflexión, por lo tanto, nos lleva a considerar que también las organizaciones son agentes morales que tienen su propio êthos, a partir de lo cual, y aunque sean personas jurídicas, les solemos conferir rasgos que corresponden a las personas físicas. Así, de acuerdo con el doctor Ramos (2009), podemos entender que «las organizaciones son también unidades vivas dotadas de inteligencia y voluntad, y también de valores. Como los seres vivos, pueden disfrutar de salud (o de una salud aceptable) y pueden enfermar» (p. 51). Aunque pueda parecerlo no se trata de diluir las responsabilidades en un maremágnum impersonal y abstracto, sino de elevar su rango puesto que adquieren una repercusión mayor cuando devienen corporativas.

Lo anterior no es incongruente con aceptar que quienes toman las decisiones sobre el ser y el devenir de una organización siempre son personas, bien individualmente o en grupo, bien por derecho propio o en representación de otros, sean directivos o subordinados. La identidad y el carácter de las organizaciones siempre son forjados - por activa o por pasivapor las personas que las integran, pero de las que resulta, al fin, un producto independiente a cualquiera de ellas. En este sentido, a la ética personal y a la ética profesional de los miembros, se suma la ética de la organización. Ángel Castiñeira (2010) dice que no es suficiente con que los miembros de una organización sean buenas personas para que la organización sea éticamente buena, sino que ha de existir una mínima ética compartida y una ética de la actuación conjunta.

Manel Peiró (2012) explica que en las burocracias profesionales (según la terminología de Mintzberg) esta clase de empleados, a menudo, entra en conflicto con la organización. Los profesionales, aunque estén en la base de la estructura organizativa, dominan una parcela del conocimiento de una forma bastante exclusiva, por lo cual, reclaman una gran autonomía en su trabajo y un bajo control de la parte gerencial de la organización. Es por esta especial relación de poder, por lo que Mendoza califica este tipo de organizaciones de «pirámides invertidas» puesto que, más que en la cúspide, el poder efectivo se encuentra en la base (Mendoza, 1995, p. 137). Pues bien, una de las aportaciones de Peiró es que «los principios y los valores de una organización burocrática no son los mismos que los principios y los valores de los profesionales, y frecuentemente colisionan» (Peiró, 2012, p. 25). Con esta advertencia queda aún más claro que, a pesar de la influencia de los profesionales en las organizaciones burocráticas (como son los Servicios Sociales), existe una independencia entre la moral exhibida por la organización y la de sus trabajadores, que a veces pueden converger pero a veces no.

\section{Las organizaciones de Servicios Sociales}

Una segunda cuestión por clarificar es iqué consideramos una organización de Servicios Sociales? ¿Se puede hablar en este ámbito de organización como algo homogéneo? Y si no fuera así, ¿qué diferencias presentan sus tipos respecto al comportamiento ético? Además, siguiendo con la distinción hecha más arriba ¿qué aporta la institución a la organización en este ámbito? Valga decir que aunque me interesa centrar el análisis en el sector específico de los Servicios Sociales (en sentido estricto), muchas de las cuestiones que siguen pueden ser aplicadas a otros sistemas del bienestar social, especialmente a aquellos de atención directa a las personas que cuentan con trabajadores sociales en sus plantillas.

Las organizaciones dedicadas a prestar Servicios Sociales son muy heterogéneas. Con es- 
to no descubrimos nada nuevo, pero es obligado revisar esta heterogeneidad, en este caso, a la luz de la reflexión sobre la ética que nos proponemos elaborar. A pesar de ello, vaya por delante que las organizaciones, por el simple hecho de funcionar como estructuras burocráticas tienen más elementos en común que diferencias en su funcionamiento.

La primera y gran clasificación es la que hace Barranco (2010) entre «organizaciones públicas, privadas y del tercer sector». Para evitar el equívoco que puede producirse entre las privadas y las del tercer sector - que también pertenecen al ámbito privado-, aquí denominaremos a las primeras empresas mercantiles. Hay que tener en cuenta además que cada uno de los tipos anteriores tiene más o menos subclases con características diferentes. De esta tipología se desprende que uno de los parámetros que más influencia debería tener para diferenciar el comportamiento ético de las organizaciones (en general) es el tipo de responsabilidad de éstas y, consecuentemente, de quien detenta la titularidad. Así, mientras unas son responsables frente al conjunto de la sociedad (las públicas), las otras responden primeramente ante los socios, accionistas o patronos.

En Servicios Sociales esto adquiere una relevancia especial porque se ha producido una cierta propensión a considerar más ética la provisión —incluida la producción — pública que la privada, lo cual, últimamente, está siendo cuestionado a causa del compromiso más radical del tercer sector; éste, a su vez, es más apreciado que el sector mercantil y goza de mejor reputación por su carácter altruista y vocacional. No puede dejar de interpelarnos este descredito en que ha caído lo público que, en gran parte, atribuimos a la interferencia del juego político (en su peor sentido) que ha subvertido los valores éticos de justicia social y de bien común, que se suponen a los que encargamos gestionar la res publica. Sin duda los Servicios Sociales sufren por parte de la clase política el mismo desprecio que otros sistemas de bienestar, y cada vez más gente entiende que esto atenta profundamente contra los mínimos valores de una ética cívica.

Es en este sentido que las organizaciones públicas que prestan Servicios Sociales no solo experimentan la tensión entre política y técnica, sino también entre los intereses partidis- tas enfrentados al bien común y a la dignidad de las personas como fin en sí mismas. A menudo, los Servicios Sociales se hallan secuestrados por intereses ideológicos y sectarios que enturbian una visión ética meridianamente diáfana de los asuntos que tratan. $\mathrm{O}$ subsisten enredados en una maraña administrativa y burocrática que adormece o desactiva la consecución de los objetivos que tan pomposamente se proclaman. Por desgracia, la dinámica cotidiana suele terminar por acomodar o expulsar las voces críticas en aras de normalizar las prácticas como un status quo amoral que impregna toda la organización.

Precisamente, por este deterioro que constamos, es necesario recuperar en cualquiera de los tres tipos de organizaciones de Servicios Sociales, un criterio crucial que nos permita, a pesar de todo, tratarlas homogéneamente y estudiar genéricamente su dimensión ética. Se trata de lo que hemos identificado como finalidad institucional. Salcedo (2010), refiriéndose a la ética de los trabajadores sociales, lo expresa como sigue: «las sociedades actuales sienten que tienen la responsabilidad de ocuparse de las personas que no pueden salir adelante por si mismas; y el trabajo social ha surgido como la profesión que se compromete a realizar esa responsabilidad social» (p. 29). Por extensión podemos decir que la complejidad de esta finalidad social ha dado pie a que la intervención se haya multiprofesionalizado y se haya instituido un sistema de atención bajo responsabilidad pública.

Estamos pues, ante la institución de la asistencia, de la atención social o de la procura de un cierto bienestar social, que constituye el hilo conductor al que deben responder todas las organizaciones de este ámbito. A esta institución se la puede identificar con nombres diversos, pero en nuestro contexto se ha impuesto el de servicios sociales (Roncal, 2011, p. 83). La institución es la razón de ser de todas las organizaciones que realizan la misma función puesto que le aportan la finalidad última, por encima de los objetivos organizativos. Roncal lo expresa de esta manera: "detrás de una organización está siempre la institución que le da su marco, que la regula y que alberga los valores del funcionamiento de la organización y de los sujetos dentro de ella, pues la institución cobra vida en las organizaciones» (ídem). 
Por lo que se refiere a la dimensión ética se trata, pues, de tres niveles interrelacionados del más concreto al más general: la ética profesional, la ética de la organización y, también, la ética institucional de la que ambas primeras forman parte. Heclo (2010) afirma que hay maneras de hacer las cosas que "constituyen una especie de moral interna que emana del hecho mismo de que la institución esté orientada a algún tipo de fin» (p.140). Entre estos niveles existe una evidente relación de subordinación, pero también de independencia, que permiten estudiarlos como tales. La institucional es la ética de los fines, la organizacional es la ética de las estrategias y la profesional es la ética de los actos. Y es imprescindible conseguir la mayor coherencia entre los tres estadios sin confundir a quien corresponde la responsabilidad ética en cada caso.

Las organizaciones privadas, a pesar de su mayor grado de discrecionalidad, o precisamente por ello, no están exentas de caer en prácticas poco éticas bien sea con los usuarios-clientes o bien con las personas empleadas. Aparte de si son consideradas con o sin ánimo de lucro, hay otras muchas variables que intervienen en una mayor o menor propensión a la observancia ética: su origen, el tipo de liderazgo, el tamaño de su estructura, su entorno, etc. Por regla general se puede apreciar que el compromiso ético es mayor en las entidades de dimensiones más reducidas, de estructuras más planas, con menos niveles jerárquicos y jefes más próximos a las bases, en las en que se fomenta el trabajo en equipo; en resumen, las que podemos llamar «entidades a la medida del ser humano», en que tanto la colaboración como el control social son mecanismos ecológicamente integrados.

Puede que algunas organizaciones de Servicios Sociales se incluyan o dependan, al menos, de dos instituciones. Es lo que pasa en aquellas que son parte de una confesión religiosa o de un grupo empresarial con intereses en otros sectores de actividad. En el primer caso nos podemos plantear $i$ es correcto exigir, a un profesional contratado por una entidad de Servicios Sociales, unas prácticas religiosas determinadas? ¿Se le podría pedir como requisito en la selección que profesara dicho credo? ¿Estaría la entidad legitimada para marcarle la orientación que debe dar a los usuarios en su trabajo profesional? Lo mismo sucedería en el caso de que fuera un grupo empresarial - como los que últimamente se han dedicado al sector de la tercera edad-. ¿Pueden obligar a un profesional a que sea cliente de otros negocios del grupo? ¿Se le podría condicionar a que hiciera algún tipo de proselitismo entre los usuarios del servicio social?

Por otra parte, las organizaciones no constituyen elementos aislados, independientes, ni son autosuficientes, sino que interactúan constantemente con otros agentes y entes organizativos; son los grupos implicados (también conocidos como stakeholders $)^{2}$. La red de relaciones incide no solo en temas de multidependencia técnica (financiera, de provisión o derivación, etc.) sino también en posicionamientos morales y perspectivas éticas en que cada parte puede tener y defender sus criterios e intereses. Lo que resulta importante y necesario cuando se quiere practicar la ética dialógica, es reconocer la potencial contribución de todos los grupos y que, en base a la colaboración, todos salgan beneficiados. Toda organización debería reconocer y asumir que «es necesario generar valor para todas las partes interesadas» (Arrieta y de la Cruz, 2005, p. 57). La importancia que esto tiene para la dimensión ética viene de considerar que no es suficiente con la legítima defensa de los propios intereses sino que, en esta nueva lógica, también se deben considerar los intereses de las partes interrelacionadas.

Cada organización tendrá que identificar quienes son los principales grupos de su entorno que la pueden afectar o ser afectados por ella. Usualmente, en el ámbito empresarial se diferencia entre los grupos implicados directos (internos) y los indirectos (externos); en el primero se incluyen a patronos, accionistas y empleados, y en el segundo al resto (Guillén, 2006, p. 285). En las organizaciones de Servicios Sociales que, a pesar de su heterogeneidad, la mayoría difieren del clásico entorno empresarial, creemos que debe darse a los usuarios igual o más primacía que a los miembros internos de

${ }^{2}$ Este término, que proviene de la empresa, en definición de Freeman es «cualquier grupo o individuo que puede afectar o puede ser afectado por el logro de los objetivos de la empresa» (citado en Arrieta y de la Cruz, 2005, p. 58) 
la organización ${ }^{3}$. Proponemos, pues, que esta detección de los grupos de interés puede organizarse en estos seis parámetros:

a) Los usuarios o clientes a los que atiende la organización, incluyendo no solo a los beneficiarios directos sino también a los familiares o personas a los que se asesora y que devienen beneficiarios indirectos.

b) Los proveedores y suministradores que requiera la organización así como los posibles prescriptores que canalizan las demandas que reciben hacia nuestra organización.

c) Las administraciones públicas con responsabilidades en el respectivo ámbito en que se ubica la organización, sin olvidar los poderes legislativo, jurídico y gubernamental.

d) Los financiadores, socios o colaboradores, según sean las fuentes de ingresos de la organización (casi siempre con repercusión en el conjunto de la sociedad).

e) Los profesionales y empleados que, desde ciertas perspectivas, se consideran «clientes internos», incluyendo los encuadrados en las entidades subcontratadas que realizan parte de los procesos de intervención social así como los voluntarios si los hay.

f) Los responsables máximos: propietarios, titulares, patronos o cargos públicos (según el tipo de organización) que, a su vez, actúan por si mismos o en representación de otros: accionistas, socios, ciudadanos.

\section{Particularidades de la ética organizacional}

El tercer grupo de interrogantes es el que se refiere al propio concepto de ética. La particularidad de enmarcar la ética en las organizaciones obliga a plantear ciertas cuestiones que tienen una relevancia especial en este ámbito: ¿Qué significa introducir la ética en las organizaciones? ¿Por qué es necesaria una ética organizativa? ¿No es suficiente con el cumplimiento de la legislación? Algunos aspectos de los que aquí se plantean pueden tener también repercusión en la ética de los profesionales, pero sostengo que deberían ser asumidos por las organizaciones, puesto que éstas son también agentes morales que pueden escoger opciones alternativas en sus actuaciones.
En palabras de Cortina y Conill (2005): «la ética de las organizaciones puede caracterizarse como la articulación, aplicación y evaluación de los valores morales consistentes y las posiciones morales de una organización, por los que se define interna y externamente, y a este proceso se le ha denominado "gestión ética total", un concepto enraizado en la comprensión de la organización como un sistema» (p. 28). Incorporar la ética a la cotidianidad de las organizaciones no significa hacer algo más de lo que normalmente se hace, sino hacer las cosas de otra manera: tomar conciencia de los valores éticos por los que la entidad quiere orientar sus decisiones y tratar de seguirlos, creando un clima ético en su interior.

La ética de una organización puede definirse desde puntos de vista diferentes, pero creo que, en último término, dichos enfoques forman un mosaico armónico que lejos de contradecirse se complementan. Cortina (1997, p. 277), aun refiriéndose a la ética empresarial, sistematiza la respuesta que dan diversos autores a la pregunta de qué es la ética de las organizaciones y dice que puede entenderse como:

a) Un modo de resolver moralmente conflictos de acción; en este sentido no es una ética negativa, de prohibiciones, sino positiva, que aconseja lo que se debe hacer y posibilita llegar a acuerdos morales racionales en un proceso de argumentación.

b) La ética que trata de las relaciones internas y externas de la organización; aquí se trata de destacar los valores positivos que se quieren y de optar por un modelo de cooperación en vez de por un modelo de conflicto.

c) Puede entenderse como una ética de la dirección y la gestión; en este enfoque se trata de reconstruir la legitimidad de la organización, entendida ésta como un conjunto de elementos que configuran un proceso organizativo.

d) Finalmente, la corriente de ética de las instituciones - que engloba las anteriores - se construiría contando con un sistema de valores en la organización, la definición de su finalidad y una cultura organizacional (sobre la que volveré posteriormente).

3 La cuestión de a quien se debe considerar miembro de una organización es otra discusión teórica que han mantenido muchos autores pero a la que ahora no nos podemos dedicar. 
La justificación de la conveniencia o necesidad de que las organizaciones cuenten con la ética en su modo de actuar se puede resumir en que es una cuestión de justicia, de solidaridad, de calidad y de confianza. Estos cuatro conceptos nos pueden ayudar a situar o a referenciar la ética en el contexto organizacional, y a ellos y a alguno más nos vamos a referir.

Se dispone de bastantes evidencias que ponen de manifiesto hasta qué punto el trato justo es una dimensión importante que afecta - entre otros stakeholders - especialmente a los clientes y a los empleados de la organización. Esto significa que cualquier afectado por una acción $\mathrm{u}$ omisión que tenga respecto a ella expectativas legítimas, tiene que ser tenido en cuenta. Martínez Sánchez et al. (2002), hacen una distinción interesante cuando dicen:

La justicia percibida por los individuos dentro de una organización se basa por un lado en la justicia que tienen los procedimientos formales que rigen las decisiones en la organización (justicia procedimental) y por otro lado en la calidad del trato interpersonal que reciben durante la ejecución de dichos procedimientos (justicia interaccional) (Martínez Sánchez, De Luis Carnicer, Pérez Pérez. y Vela Jiménez, 2002, p. 123).

En la literatura sobre ética, la solidaridad aparece más diluida, pero aun así consideramos que se encuentra subsumida, tanto en el enfoque de colaboración de los stakeholders que hemos comentado anteriormente, como - sobre todo en las empresas privadas mercantiles - en lo que representa la responsabilidad social corporativa ${ }^{4}$. La evolución de dicho concepto (Arrieta y de la Cruz, 2005) ha llevado a conferirle un carácter global «que afecta a la totalidad de los elementos que integran el conjunto de la política empresarial: aprendizaje permanente; organización del trabajo; igualdad de oportunidades; inclusión social; desarrollo sostenible; previsión y gestión del cambio; protección de los derechos humanos, etc.» (p. 52). Aunque a veces se instrumentalice como un simple «efecto cosmético», la co- herencia moral de las organizaciones debe salir también fuera y proyectarse en su inserción social, en su aportación al bien común contribuyendo al desarrollo social de las comunidades en las que operan.

Últimamente, la ética se ha vinculado a los temas de calidad de los bienes y servicios. Así lo vemos incluso en las propias leyes de Servicios Sociales ${ }^{5}$. La perspectiva actual de calidad total persigue una mejora continua en todos los parámetros organizativos $\mathrm{y}$, aunque en los modelos de gestión de la calidad no se explicita directamente la ética, se puede entender incluida, más o menos, en todos los instrumentos (normas ISO, SGE 21, modelo EFQM, etc.). Según Barbero (2008):

La gestión de la calidad no es ajena a la gestión ética. Incorporar este tipo de referencias y profundizar en la dirección por valores nos permitirá avanzar en coherencia tanto en nuestra intervención con las personas destinatarias como en la vida interna de la organización, en el modo en que nos organizamos y gestionamos nuestros recursos económicos, nuestras relaciones, etc. (p. 15).

Finalmente, la confianza no sólo es imprescindible en la relación de ayuda que establecen los profesionales con los usuarios, sino que es también un valor del conjunto de la organización prestadora de servicios.

La confianza es una parte importante del capital ético, de ese activo que cuando impregna las relaciones con trabajadores, instituciones políticas, gobiernos, consumidores, proveedores, cuando se ha adquirido, jugada tras jugada, al no defraudar las expectativas, es productivo, tanto directa como indirectamente (Cortina y Conill, 2005, p. 24).

El buen comportamiento genera confianza mientras que el comportamiento éticamente reprobable la destruye $\mathrm{y}$, además, si una organización reiteradamente no reconoce lo que se hace mal, pierde credibilidad y destruye la confianza en ella depositada. Como dice Villafañe (2004) el concepto «reputación» se refiere al

${ }^{4}$ La Comisión de las Comunidades Europeas en el Libro Verde de la Responsabilidad Social de las Empresas (2001) la define como: «la integración voluntaria, por parte de las empresas, de las preocupaciones sociales y medioambientales en sus operaciones comerciales y sus relaciones con sus interlocutores».

${ }^{5}$ En la ley 12/2007 de servicios sociales de Catalunya, dentro del título VIII: de la calidad de los servicios sociales, dedica el artículo 86 a la deontología profesional. En el apartado 2 se dice: los deberes relativos a la deontología profesional se han de incluir en los criterios de calidad (...). 
juicio, positivo o negativo, que se hace del comportamiento corporativo de la organización.

La organización que actúa bien en sentido ético, se hace digna de confianza en este ámbito. Y la confianza, como la reputación (o la imagen, en un plano más superficial), constituyen juicios de valor realizados por terceros, que son siempre consecuencia, de modo que se puede incidir en su fuente, pero no siempre asegurar que se produzca como resultado final» (citado en Guillén, 2006, p. 309).

Por último, encontramos que la ética de las organizaciones también se incluye como parte de la cultura organizativa y del clima organizacional. Tratándose de un elemento de análisis, el estudio de la cultura puede resultar un lugar adecuado para centrar también la atención sobre la cultura ética, tanto de los profesionales y empleados como de la propia organización ${ }^{6}$. Según Guillén, «los elementos de la cultura que caracterizan a cada organización pueden ser factores que faciliten la aplicación de políticas de cambio hacia la ética o, por el contrario, un obstáculo para ellas» (p. 238). La cultura organizacional se entiende como el modo habitual de pensar, sentir y actuar la organización en su conjunto, siendo una parte observable y otra no observable (valores, creencias, presunciones básicas) que deben apreciarse indirectamente e interpretativamente. Al igual que la cultura, la ética es también un elemento dinámico y plural, por ello, Hortal dice que «la ética de una organización debe entenderse no como un hecho objetivo y estático que se impone por parte de quienes detentan el poder en la organización, ni tampoco se excluye que en la misma organización puedan coexistir diversas subculturas (profesionales o empresariales)» (Hortal, 2002, p. 79).

El concepto de cultura está íntimamente relacionado con el de clima organizacional aunque este segundo se circunscribe más a lo que también se conoce como ambiente laboral. Se trata de la percepción que tienen los profesionales y empleados en relación a las condicio- nes en que realizan su trabajo y en cómo son las relaciones que en él se establecen. Aquí también existe un peso importante de la ética aplicada a la política laboral para con los empleados, al cuidado de la motivación y satisfacción en el trabajo, etc.

Atendiendo a la calidad ética de estas dos dimensiones: la interna del clima y la cultura organizacional, y la externa de la confianza y la reputación, podemos concluir con los autores que,

El clima ético se crea en una organización cuando sus miembros perciben que, en los distintos niveles, las decisiones se toman atendiendo a los valores éticos de la misión de la entidad; esta percepción crea dos tipos de beneficio a la vez: cohesión interna en torno a los valores de la organización [...] y una imagen externa positiva, una buena reputación, porque los afectados por la actividad de la organización detectan su buen funcionamiento y les inspira confianza» (Cortina y Conill, 2005, p. 28).

Otra cuestión que conviene discernir es que no basta con que una organización se ajuste a la ley para que por ello podamos considerarla ética. Sin embargo, esta idea acostumbra a estar muy (iinteresadamente?) extendida en determinadas organizaciones tanto públicas como privadas. Así, de considerar que toda acción que no sea punible jurídicamente es lícita, se pasa a pensar que cualquier organización tiene un comportamiento ético mientras no se detecte, se denuncie y se demuestre lo contrario. Se pasa por alto que las vulneraciones de la ética no suelen estar convenientemente definidas, controladas e, incluso, mal consideradas en todas las organizaciones como para que lleguen a ser denunciadas. Pero hay que insistir en que una cosa puede ser legal y a la vez moralmente reprobable.

\section{Rodríguez-Arana (1997) opina que}

Debemos partir, pues, de una visión de la codificación ética como complemento de la tipificación legal de las conductas y como cláusula de garantía, respecto de los conflictos humanos que se suscitan en el ámbito de la actividad

${ }^{6}$ Edgar Schein es uno de los máximos exponentes del estudio de la cultura organizacional que define como «el conjunto de presunciones básicas - inventadas, descubiertas o desarrolladas al ir aprendiendo a enfrentarse con sus problemas de adaptación externa e integración interna-, que hayan ejercido la suficiente influencia como para ser consideradas válidas y, en consecuencia, ser enseñadas a los nuevos miembros como el modo correcto de percibir, pensar y sentir esos problemas» (1988) La cultura empresarial y el liderazgo. Barcelona, Plaza \& Janés. 
administrativa y a los que el Derecho no alcanza a dar una respuesta adecuada (p. 32).

Se trata de dos niveles diferentes, el primero (el legal), de obligado cumplimiento y el segundo (el moral) donde hay que incluir otros compromisos autoimpuestos que se refieran a conductas acordes con valores como la dignidad humana así como un concepto de justicia más allá de lo judicial.

Y aún hay más. En esta compleja inter-independencia entre el Derecho y la Ética, hay incluso quien afirma que "a veces la ética te obliga a no cumplir la ley: es decir, la desobediencia civil» (Oliveras, 2010, p. 97) o a hacer objeción de conciencia, como propugnó la Sociedad Española de Medicina de Familia y Comunitaria frente a la regulación que dejaba sin asistencia sanitaria a las personas inmigrantes que se encontraban irregularmente en España. También en Servicios Sociales a menudo se dan situaciones dilemáticas en que, o bien se incumplen determinados preceptos para, en la práctica, corregir aquello que a todas luces producirá efectos perniciosos en los usuarios o, por el contrario, se sigue a pies juntillas la legislación y se deja que la «maquinaria burocrática» produzca una flagrante iniquidad.

Esto enlaza de alguna manera con la diferencia clásica entre la ética de la convicción y la ética de la responsabilidad (debemos a Max Weber esta distinción que incluye en El politico y el científico). Mientras la primera considera las acciones en sí mismas, sin tener en cuenta las condiciones en qué se realicen ni las consecuencias que se devengan, la segunda, en cambio, sí que las tiene en cuenta; una da prioridad a los principios y valores (por ejemplo, no mentir) mientras la otra se basa en los resul- tados que producirían las alternativas de acción. Ahora bien, aferrarse sólo a las convicciones conlleva el peligro del fundamentalismo, así como guiarse exclusivamente por las consecuencias supone caer en un utilitarismo desnortado.

Pero «hablar de ética significa que, cuando ejecutamos una decisión, debemos pensar en qué impacto social tendrá [...] en las entidades sociales se genera una tensión muy interesante entre nuestras convicciones y nuestras consecuencias» (Castiñeira, 2010, p. 91). A veces las organizaciones (y sus profesionales) se ven obligadas a flexibilizar sus principios maximalistas para conseguir resultados que, sin traicionar los valores, minimicen también las consecuencias indeseables para la población y la propia organización. A esta postura híbrida, Cortina la llama responsabilidad convencida (1997, p. 273).

Como conclusión de esta primera parte podemos afirmar que las organizaciones son agentes morales y así se las debe considerar al juzgar las decisiones corporativas. Entre las de Servicios Sociales, unas participan del descredito de lo público y otras del aprecio por su respuesta de proximidad. En la valoración de cualquiera de ellas se debe discernir el nivel institucional del organizativo y no hay que olvidar el papel que juegan los stakeholders en la modulación de sus intereses con los valores éticos. Finalmente, la conveniencia de la ética organizacional se explica en cuatro conceptos: justicia, solidaridad, calidad y confianza a los que vinculamos con la cultura y el clima de la organización; en cualquier caso más allá de la legalidad estricta y pretendiendo alcanzar cierto equilibrio entre la ética de la convicción y de la responsabilidad.

\section{Referencias bibliográficas}

Arrieta Heras, B., y De la Cruz Ayuso, C. (2005). La dimensión ética de la responsabilidad social. Bilbao: Universidad de Deusto.

Barbero Blanco, I. (2008). Sistemas de gestión de la calidad en el tercer sector de acción social: situación actual y propuestas de futuro. Zerbitzuan, 43, 7-18.

Barranco Expósito, C. (2010). Retos del bienestar en los ámbitos del Trabajo Social: potenciar la responsabilidad social en las organizaciones públicas, privadas y del tercer sector. En A. Rodríguez Monge y E. Raya Lozano (coords.), Una Europa social y plural. Actas del VII Congreso de Escuelas Universitarias de Trabajo Social. Granada: Universidad de Granada.

Castiñeira Fernández, A. (2010). L'Ėtica de les organitzacions socials. En Els reptes ètics de la intervenció social (pp. 88-95). Girona: Observatori d'ética aplicada a la intervenció social.

Cortina Orts, A. (1997). Ética aplicada y democracia radical. Madrid: Tecnos. 
Cortina, A. y Conill, J. (2005). Ética, empresa y organizaciones sanitarias. En P. Simón, Ética de las organizaciones sanitarias. Nuevos modelos de calidad. Madrid: Triacastela.

Coulshed, V. (1998). La gestión del trabajo social. Barcelona: Paidós.

Douglas, M. (1996). Cómo piensan las instituciones. Madrid: Alianza Editorial.

Guillén Parra, M. (2006). Ética en las organizaciones. Construyendo confianza. Madrid: Pearson Educación.

Heclo, H. (2010). Pensar institucionalmente. Madrid: Paidós.

Hortal Alonso, A. (2002). Ética general de las profesiones. Bilbao: Desclée De Brouwer.

Margalit, A. (1997). La sociedad decente. Barcelona: Paidós.

Martínez Sánchez, A., De Luis Carnicer, M.P., Pérez Pérez, M. y Vela Jiménez, M. J. (2002). El potenciamiento de los equipos de trabajo: análisis de sus factores influyentes. Acciones e Investigaciones Sociales, 15, 109-129.

Mendoza, X. (1995). La funció directiva en les organitzacions de professionals. En Les àrees de serveis personals: anàlisi del procés i propostes de futur (pp. 135-148). Barcelona: CIFA, Diputació de Barcelona.

Oliveras, A. (2010). Puntualitzacions a aspectes sorprenents. En Els reptes ètics de la intervenció social (pp. 96-98). Girona: Observatori d'ètica aplicada a la intervenció social.

Peiró, M. (2012). La lógica generencial. Cuadernos de la Fundació Victor Grífols i Lucas, : La ética en las instituciones sanitarias: entre la lógica asistencial y la lógica gerencial, 28, 23-33.

Ramos Montes, J. (2009). Autonomia i vulnerabilitat en les persones amb discapacitat intel·lectual. Quaderns d'Acció Social i Ciutadania, 6, 48-53.

Rodríguez-Arana Muñoz, J. (1997). Ética y empleados públicos. Gestión y Análisis de Políticas Públicas, 9, 29-34.

Roncal Vargas, C. (2011). La ética en las organizaciones de servicios sociales y en los equipos de trabajo. En T. Zamanillo, Ética, teoría y técnica. La responsabilidad política del trabajo social (pp. 81-112). Madrid: Talasa.

Salcedo Megales, D. (2010). Los fundamentos normativos de las profesiones y los deberes de los trabajadores sociales. Trabajo Social Global [en linea]: Recuperado de http://www.hipatiapress.info/hpjournals/index.php/tsg/search/titles), 10-38.

Valero Matas, J. A. (2008). Las instituciones y organizaciones sociales. Madrid: Pirámide. 